\title{
Readiness for First-In-Human Neuromodulatory Interventions
}

\author{
Iris Coates McCall@D, Nicole Minielly, Allison Bethune, Nir Lipsman, \\ Patrick J. McDonald, Judy Illes (iD
}

\begin{abstract}
Background: Novel neurointerventions present innovative therapeutic approaches to a range of treatment-refractory disorders. We sought to characterize factors that inform and define translational readiness for first-in-human (FIH) neuromodulatory trials. Methods: We used a two-part methodology involving a scoping review of the biomedical literature on the readiness of FIH trials for both neurological and non-neurological applications, and semi-structured interviews with stakeholders about decision-making for neuromodulation using magnetic resonance-guided focused ultrasound as a case example. Results: One hundred and thirty factors relevant to FIH readiness were identified in the scoping review. Trial design, adequacy of preclinical evidence, and risk were ubiquitous across biotechnologies. Target organ, target function, and inadequacy of animal models were dominant in the neurointervention literature. Interview results on the relative importance of these factors reveal divergent values, priorities, and understandings both between patients and clinicians and between patients affected by different conditions. Conclusion: Readiness of neurotechnology for FIH trials is defined by a multitude of interacting factors that pertain to clinical and nonclinical priorities, perceptions, and values.
\end{abstract}

RÉSUMÉ : Niveau de maturité translationnelle en lien avec les premières interventions neuro-modulaires chez l'être humain. Contexte : Les nouvelles techniques de neuro-intervention présentent des approches thérapeutiques innovantes dans le cas d'une série de troubles neurologiques réfractaires à un traitement. Nous avons donc cherché à caractériser les facteurs pouvant nous renseigner au sujet du niveau de maturité translationnelle (translational readiness) des premiers essais neuro-modulaires chez l'être humain. Méthodes : Notre méthodologie s'est divisée en deux volets : d'une part, un examen exploratoire de la littérature biomédicale quant au niveau de maturité des premiers essais chez l'être humain en vue d'applications neurologiques et non-neurologiques ; d'autre part, des entrevues semi-dirigées avec les parties prenantes du domaine en ce qui a trait au processus de décision en lien avec la neuro-modulation. À titre d'exemple, nous avons évoqué le traitement par ultrasons focalisés sous guidage IRM (magnetic resonance-guided focused ultrasound). Résultats : Au total, 130 facteurs se rapportant au niveau de maturité de ces premiers essais chez l'être humain ont été identifiés dans le cadre de notre examen exploratoire. La conception des essais, l'adéquation des preuves cliniques et le risque sont des facteurs du domaine des biotechnologies qui se sont révélés omniprésents. Cibler des organes, cibler des fonctions et l'inadéquation des modèles animaux se sont avérés par ailleurs des facteurs dominants dans la littérature portant sur la neuro-intervention. Nos résultats d'entrevues ont aussi mis à jour des valeurs, des priorités et des compréhensions divergentes à la fois entre les patients et les cliniciens mais aussi entre les patients atteints de diverses affections. Conclusion : Le niveau de maturité des neuro-technologies en vue de premiers essais chez l'être humain peut se définir par une multitude de facteurs qui interagissent et qui se rapportent à des priorités cliniques et non cliniques mais aussi à des perceptions et à des valeurs.

Keywords: Neuroethics, First-in-Human, Neuromodulation

doi:10.1017/cjn.2020.113

Can J Neurol Sci. 2020; 47: 785-792

\section{INTRODUCTION}

Ground-breaking technological advancements in the neurological sciences are yielding innovative therapies for previously intractable disorders of the central nervous system and mental health conditions. Novel neurosurgical procedures and implants have been proposed for psychiatric, neurologic, and oncological conditions, and neuromodulation that intervenes on brain circuitry to stop, start, and interrupt systems at the root of brain disorders is at the driving edge of this innovation. The transition from preclinical research to first-in-human (FIH) trials, however, is challenged by a relative absence of information on safety, efficacy, and risk. ${ }^{1}$ Unlike pharmaceuticals that have regulatory minima for evidence of safety and efficacy required prior to approval, regulations describing how novel, non-pharmaceutical interventions should be tested are lacking. This is widely noted in the literature as a barrier to innovation, and a failure of the regulatory process, especially in surgical fields. ${ }^{2-5}$

Well-established benchmarks of acceptable levels of preclinical data in pharmaceutical development enable translation on that landscape to proceed ethically. ${ }^{6}$ Even so, Phase 1 clinical trials are the riskiest given the inherent lack of pre-existing data necessary for the ethical justification of research with human subjects. ${ }^{7,8}$ Furthermore, phase 1 FIH trials are designed to test for safety and tolerability, not efficacy. For this reason, there can

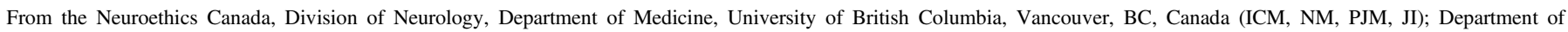

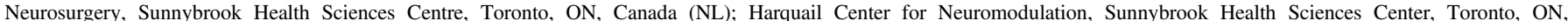

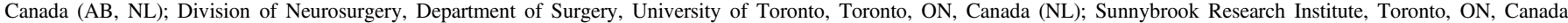

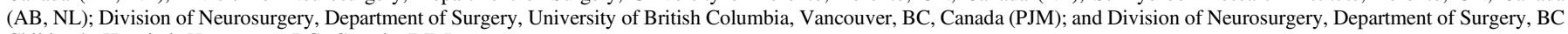
Children's Hospital, Vancouver, BC, Canada (PJM)

Received December 22, 2019. Final Revisions Submitted May 26, 2020. Date of Acceptance May 29, 2020.

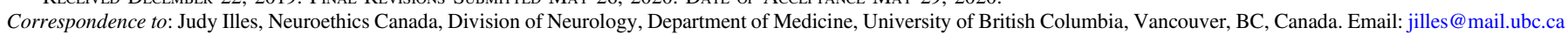


be no reasonable expectation of direct benefit to the participant from trial participation. It is a cornerstone principle of bioethics that there must be sufficient evidence to expect benefit in the long term, if not short term. FIH trials are unlikely to benefit individual participants, and therefore, any significant risk to individuals seems difficult to justify. The justification that nothing else is available may not be adequate in this case. Therefore, the riskbenefit assessment fails to support enrollment in FIH trials. ${ }^{9,10}$

Much attention has been paid to the ethical and technical difficulties of overcoming this hurdle. ${ }^{9,11,12}$ Novel surgical innovation, however, presents a unique case for translation. While novel, untested surgeries of unknown efficacy can be morally permissible in the context of a best care option for a patient for whom no other therapeutic approach has been or is likely to be successful, such cases often fall outside the realm of ethical research oversight mechanisms, and therefore outside the purview of standardized protocols and guidance. ${ }^{13}$ This lack of guidance is present for many innovative and experimental treatments, but especially so for novel neuromodulatory interventions that often include a mixture of novel surgical procedures, medical devices, and therapeutic agents that span several different clinical domains all with different oversight and regulatory guidelines. While not necessarily specific to the brain - other novel interventions such as gene transfer can also span clinical designs and domains - many people perceive the brain to be exceptional because of its central role in personhood, sense of self, agency, and autonomy. ${ }^{14-16}$ This makes the ethical and regulatory concerns all more weighty for neuromodulatory interventions. Past research has offered recommendations for enhancing regulation surrounding surgical innovation $^{17}$ and discussed the importance of balancing technological innovation with the minimization of risks to patients. ${ }^{18}$

Magnetic resonance-guided focused ultrasound (MRgFUS) is a novel neuromodulatory intervention that involves concentrating a large number of ultrasound beams on a small area in the brain as informed by near real-time neuroimaging of the target. Highfrequency MRgFUS heats up targeted tissue enough to ablate it. High-frequency MRgFUS has been proposed as a scalpel-free equivalent to more traditional ablative brain surgeries that require scalp incisions, holes in the skull, and the transgression of brain tissue to reach the target. Low-frequency MRgFUS can temporarily open the blood-brain barrier (BBB) by activating injected microbubbles at a certain frequency. The goal is to allow novel therapeutics with molecules too big to pass through the BBB to enter the brain and reach their target. World first trials using MRgFUS are currently being tested for oncological, psychiatric, and neurodegenerative conditions. ${ }^{19-21}$

\section{Methods}

We applied a two-part mixed-methods protocol to explore how FIH biotechnologies can inform FIH trials specifically for innovation for neurologic and psychiatric conditions. We conducted a scoping review ${ }^{22}$ of the literature on FIH neurotechnologies and biotechnologies broadly, and a set of qualitative semi-structured interviews with participants and clinicians involved in $\mathrm{FIH}$ MRgFUS trials as a case example.

\section{Scoping Review}

We generated a comprehensive database of English-language peer-reviewed literature for analysis by applying search terms (e.g., FIH, neuro*, readiness) in the form of medical subheadings, free-text words, and appropriate truncations pertaining to the concepts of FIH trials for medical technologies, risk, readiness, translation, and ethics to PubMed, EMBASE, and Google Scholar. Terms were customized as necessary for the different databases to maximize the sensitivity of the search. All databases were searched from their inception without date or geographic restrictions, and all studies referencing FIH novel medical technologies were included, regardless of study design or methodology. Returns were manually curated for relevance and duplicates removed using dedicated software. Disciplinary categories emerged from the search and were not defined a priori. Pharmaceutical studies were excluded.

We used qualitative methods ${ }^{22}$ to identify main categories and subcategories of factors relevant to FIH decision-making in the final set of relevant unique papers, generating a factor pedigree for each. For analysis, we clustered neuro* papers (e.g., neuroscience, neurotechnology, neurology, and neurosurgery) and non-neuro papers into two separate groups. A factor - the central unit of analysis - was coded only once per paper regardless of the extent of related discussion. Given unequal numbers of papers in each group, findings were converted to proportions, with frequency of factor citation as the numerator and number of papers in that group as the denominator. We analyzed the data using descriptive statistics and provide exemplary quotes to deepen the quantitative findings where appropriate.

\section{Semi-Structured Interviews}

We conducted a single-site case series of interviews with patients and clinicians involved with FIH MRgFUS trials at a major Canadian research hospital. Purposive sampling was used to recruit participants to the study. Interviews were conducted with patients who opted for a novel MRgFUS neurointervention over a more established procedure or no treatment, and surgeons, oncologists, and psychiatrists involved with FIH MRgFUS trials. Open-ended questions probed perceptions of risk, readiness, and invasiveness of novel neurointerventions. All interviews were audio-recorded, transcribed, and made software ready for analysis. We applied qualitative description and direct content analysis to the data, using an a priori coding scheme informed by the results of the scoping review, ${ }^{23-26}$ and others emerging organically from the interviews. Identification of major and minor categories of factors was based on theoretical relationships between the factors that formed the unit of analysis.

Sample of Interview Questions - Clinicians

- How do you explain the procedure to the patients?

- How do you personally evaluate a surgery for its readiness for translation?

- What factors do you take into consideration when determining if something is ready for FIH trials?

Sample of Interview Questions - Patients

- What kind of information did you receive about the different options available to you?

- Of all the different attributes of the intervention, which do you think was most important to you?

- What about the procedure made you interested in it? 


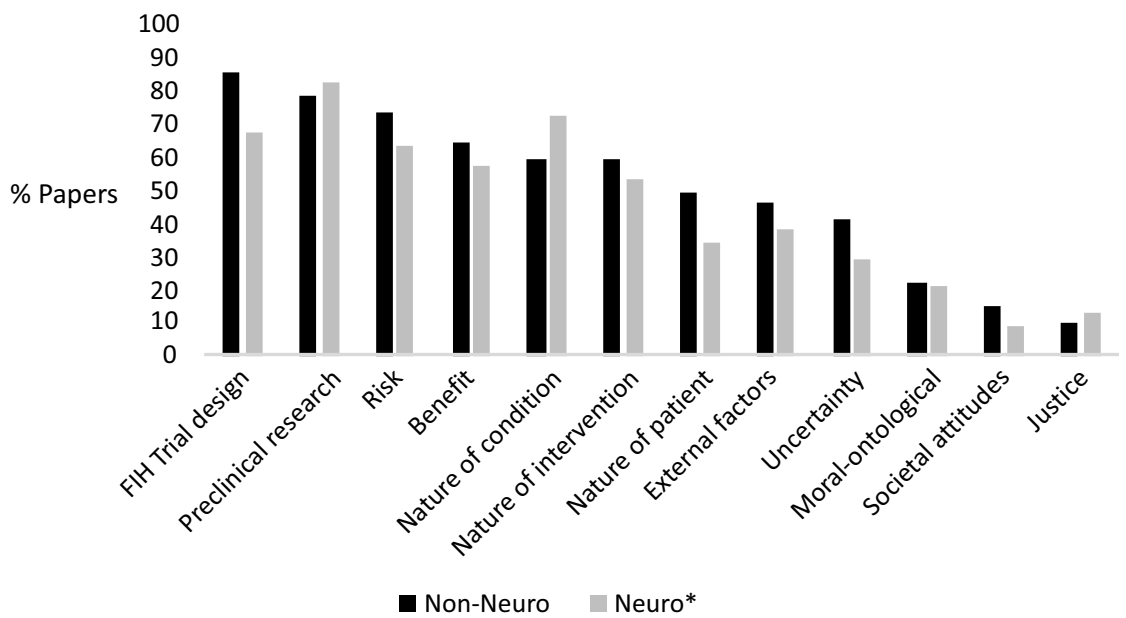

Figure 1: Frequency of top-tier factors identified in the literature.

\section{RESULTS}

Using the Cohen's kappa test, we report high intercoder reliability with a coefficient of 0.83 for the scoping review and 0.85 for the analysis of the interviews.

\section{Scoping Review}

A total of 224 individual papers met inclusion criteria. The non-neuro papers spanned the disciplines of general translational research $(n=55)$, genetic engineering $(n=6)$, nanotechnology $(n=7)$, obstetrics $(n=3)$, stem cells $(n=38)$, surgery $(n=39)$, synthetic biology $(n=4)$, and tissue engineering $(n=4)$. One hundred and twelve (112) were included in the neuro* group. One hundred and thirty (130) unique factors relevant to the decision to proceed with a FIH trial were identified across the 224 papers. These factors fell into 12 dominant thematic categories (Figure 1).

The three overarching categories of factors that dominated the non-neuro FIH literature were FIH trial design, adequacy of the preclinical research supporting the intervention, and risk. Each major factor also had constituent second- to third-tier subfactors (Figure 2).

\section{FIH Trial Design}

The dominant top-tier factor of FIH trial design was discussed in $86 \%$ of the non-neuro literature and $65 \%$ of the neuro* literature. It consisted of 26 subfactors as shown in the factor pedigree in Figure 2.

The most highly cited subfactor relevant to translation was choice of study population, mentioned in $50 \%$ of non-neuro papers and $36 \%$ of neuro* papers. Several papers argued that only the sickest patients should be involved in trials because they have "less to lose" than less affected patients and therefore a more favorable risk-benefit profile:

Traditionally, the patients approached for participation in FIH trials are usually those with severe or advanced disease, for whom there are no good treatment choices available (either because all standard treatments have failed or because no good standard treatment exists). ${ }^{27}$
By contrast, other papers favored involvement of the healthiest patients because they are likely to have the fewest complications:

Enrolled phase 1 subjects are generally healthy volunteers, or if they have some clinical pathology... their condition is relatively stable, so that participation in a short clinical study does not pose a serious health threat. ${ }^{28}$

Fifty percent of non-neuro papers and 30\% of neuro* papers discussed how determinations of readiness for translation into FIH trials was impacted by whether the goal of the trial was to simply establish the safety of the intervention, or if it was to determine the efficacy of the intervention for its intended clinical purpose. Dosing was described in $32 \%$ of non-neuro papers and $26 \%$ of neuro papers.

\section{Adequacy of Preclinical Evidence}

Discussion of the adequacy of preclinical evidence for determining the readiness for FIH trials in the biotechnology literature focused on the subfactors animal models (62\% of non-neuro literature; $71 \%$ of neuro*), the quality of the preclinical research, including internal and external validity (41\% non-neuro; $40 \%$ neuro*) and scientific rationale (29\% non-neuro; $46 \%$ neuro*).

Dresser (2012) writes that adequate preclinical data are:

an ethical and regulatory requirement for FIH nanotrials,

and that

without solid preclinical evidence, reviewers cannot determine whether the benefits a trial is expected to produce are sufficient to justify the anticipated risks to FIH trial participants... ${ }^{11}$

The factor pedigree for adequacy of preclinical evidence is shown in the online supplemental material. Discussion about animal models in the neuro* literature focused on their inability to mimic changes in mood or cognition in certain neurologic conditions, that models often mimic only one aspect or symptom of the condition, and that limited longevity of animals fails to mimic disease progression for neurodegenerative diseases: 


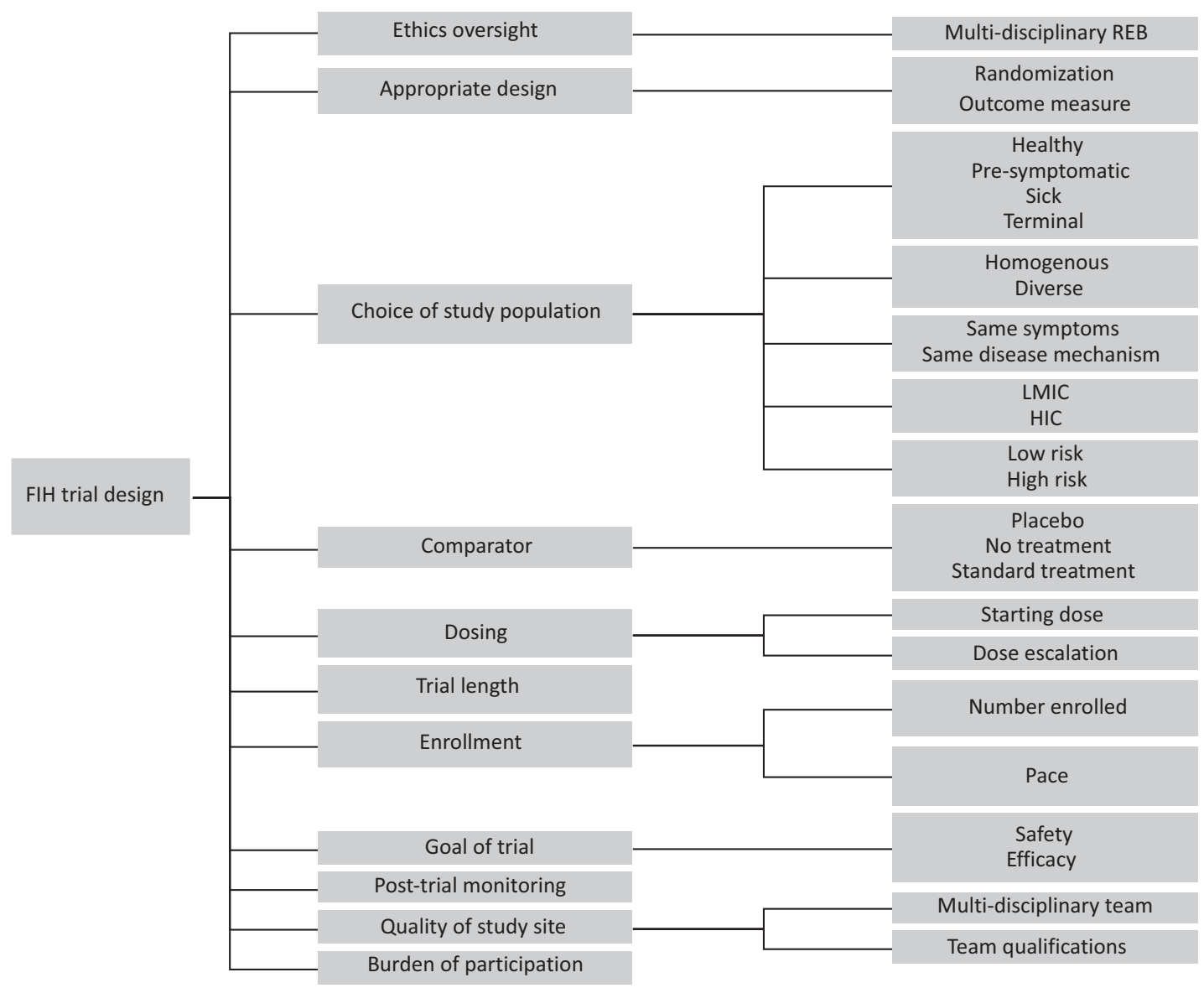

Figure 2: Factor pedigree for FIH trial design.

[...] [t]he reasons behind the lack of beneficial effects of those putative therapies in humans is related to limitations of the mouse models per se, in particular with respect to their inability to faithfully recapitulate pathophysiological features observed in the human disease. ${ }^{29}$

\section{Risk}

Subfactors under the top-tier factor risk fell into three categories: direct physical risks to the patient (e.g., side effects, safety, and latency of potential negative side effects); indirect risk (e.g., psychological, financial, social risks); and societal risk (e.g., potential loss of public trust or support for the intervention, or harm to the environment in the case of genetic research). We found that the complexity of calculating risk for FIH readiness was a further consideration within these traditional categories:

$[\ldots]$ now in life and in all sectors of human activity, (...) we encounter risk that is in a sense calculable. And when you are working within a realm that is highly novel like gene therapy or novel stem cells approaches or nanomedicine, you are dealing with circumstances where it is very difficult to calculate the risk. ${ }^{30}$

Ambiguity in novel intervention studies takes multiple forms... translational studies present ambiguous risks as well as ambiguous gains (with the latter dividing into ambiguous social and direct benefits)... In translational trials, ambiguity involves both outcomes as well as probabilities. Translational trials are thus seemingly saturated in ambiguity. ${ }^{31}$

This uncertainty also emerged as a top-tier factor, discussed in $44 \%$ of non-neuro papers and $29 \%$ of neuro* papers:

Early human trials are conducted at the point of highest uncertainty in clinical development of an intervention. ${ }^{7}$

Among the top-tier factors, nature of the condition most distinctly distinguished the neuro* (72\%) and non-neuro (59\%) literatures representing an increase in discussion of $13 \%$. Distinguishing factors nested under the top-tier factors were scientific rationale, target function of the intervention, target organ, use of animal models, and illness prevalence, which were each discussed over $10 \%$ more in the neuro* over non-neuro literature.

Several factors relevant to determining FIH intervention readiness in non-neuro biotechnologies were absent from the neuro* literature. Of 143 non-neuro papers, financial benefit was mentioned in both the general and stem cell papers $(3 \%$ of all non-neuro literature); religious considerations were mentioned in the context of stem cells and synthetic biology (3\% of non-neuro literature); clinician willingness to perform novel interventions was mentioned in the surgical literature $(1 \%$ of non-neuro literature), and largely attributed to the potentially negative 


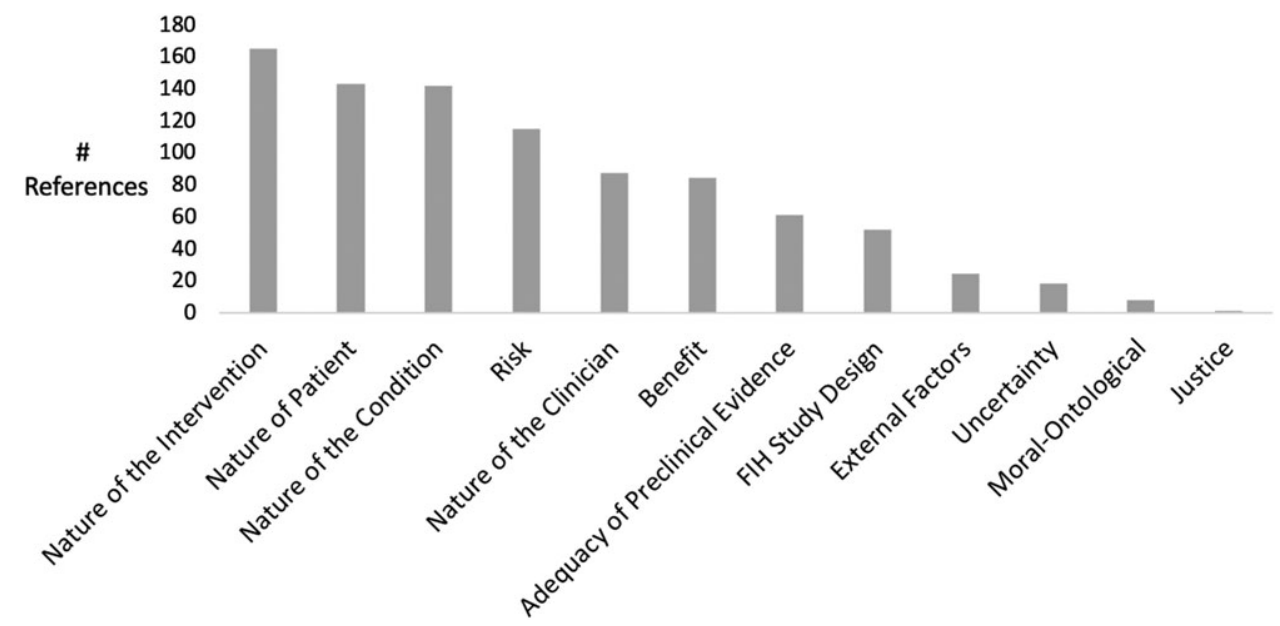

Figure 3: Frequency of top-tier factors in interviews.

impact on performance statistics. The surgical literature also mentioned legal liability $(<1 \%$ of non-neuro literature) and urgency ( $3 \%$ of non-neuro literature). Finally, public fear of the novel intervention emerged in two instances in the synthetic biology literature (1\% of non-neuro literature) attributed, in part, to concerns about transhumanism.

\section{Semi-Structured Interviews}

We conducted 17 interviews: 12 with patients $(n=6$ neurodegenerative conditions, $n=6$ psychiatric conditions) and 5 with clinicians ( $n=2$ surgeons, $n=2$ oncologists, $n=1$ psychiatrist). Conditions represented were amyotrophic lateral sclerosis (ALS, $n=4$ ), Alzheimer's disease (AD, $n=2$ ), obsessive compulsive disorder (OCD, $n=3$ ), and major depressive disorder (MDD, $n=3$ ). Interviews ranged from $10 \mathrm{~min}$ to $1: 45 \mathrm{hr}$ for a total of $10 \mathrm{hr} 51 \mathrm{~min}$ of recordings and focused on perceptions of risk, readiness, and the decision-making process for FIH trials. Venue and scheduling constraints contributed to the variability of interview lengths. Some interviews were limited in length as they were conducted between patients' and clinicians' busy schedules at the clinic. In the qualitative analysis, we took this variability into account by assuring that themes repeated by one person were only accounted for as single occurrence. The results, therefore, are cross-cutting and not dominated by any one participant. Differences in interview lengths were not correlated with type of participant (i.e., clinician vs patient). The 12 top-tier factors representing the 130 factors identified from the literature analysis formed the basis of an initial codebook that was then refined to analyze the interviews.

Dominant top-tier factors identified in the interviews were nature of the intervention ( $n=165$ references, $17 / 17$ participants), nature of the patient ( $n=143$ references, 17/17 participants), and nature of the condition ( $n=142$ references, $17 / 17$ participants) (Figure 3).

\section{Nature of the Intervention}

The factor nature of the intervention included the subfactors invasiveness of the procedure (49 references, 17/17 participants), time considerations such as duration and latency of effects (41 references, 12/17 participants), and the frequency of the intervention (21 references, 12/17 participants). Clinicians and patients reported different perceptions of MRgFUS invasiveness: while $100 \%(5 / 5)$ of the clinicians described the procedure as invasive or minimally invasive, $50 \%(6 / 12)$ of the patients described the procedure as non-invasive or "not real surgery":

I like to make sure they realize and appreciate fully that this is invasive, and this is irreversible damage to the brain.

Clinician C3, Psychiatrist

[...] it's not surgery... So it's not as if you're being cut open. I don't think it's an invasive procedure.

Patient P5, AD Patient

\section{Nature of the Patient}

Under the second most highly cited top-tier factor, nature of the patient, clinicians discussed how patient comprehension was a key concern for them in deciding whether or not to move forward with a trial (56 references, 16/17 participants):

I don't want patients going through with any illusions about scalpel-less surgery, that it's somehow not a surgery. So, I usually hammer that home, fairly hard, to make sure I've got full comprehension.

\section{Clinician C3, Psychiatrist}

Although patients reported that they felt adequately informed by the clinical team prior to the procedure, they also reported augmenting their understanding of the procedure with independent research. Also under nature of the patient, patients identified desperation (13 references, 8/17 participants) and trust in the physician (11 references, $5 / 17$ participants) as major subfactors that influenced their decision-making. 


\section{Nature of the Condition}

The third most cited top-tier factor nature of the condition (142 references, 17/17 participants) was dominated by discussions of severity (51 references; $13 / 17$ participants). Both clinicians and patients expressed that severity was correlated with their willingness to participate in a FIH trial and that they would not have considered a FIH trial if the condition was not currently or foreseeably severe:

... I wasn't [concerned about the risks] and I think the reason for that is that... knowing where this disease is going to go and it's going to be inevitable, that I was prepared to take the risk of something going wrong because the end result isn't good anyway.

\section{Patient P5, AD Patient}

Thirty-eight references to the availability of alternative treatments (16/17 participants) highlighted how the lack of other treatment options further influenced participation decisions in trials of unknown efficacy. Twelve of 17 participants discussed how the ineffectiveness of standard care was also determining factor (31 references).

\section{Emergent Themes}

Patient motivation for participating in the FIH trials emerged as a key difference between patient groups - a major finding. Six of six patients with neurodegenerative disorders reported that they would participate in the trial even if there was no chance of direct benefit to them, and would do so to help future patients:

Anything that I can do-even if it's not in time for me - but anything I can do to advance the science and be part of that process to get us to the day when this thing is either curable or manageable, you know, I would like to do my part if I can.

\section{Patient P6, ALS Patient}

By contrast, only one of the patients with a psychiatric condition would consider participating in a FIH trial for altruistic reasons:

Not for something this invasive... If I had thought the chances were very slim, I wouldn't have done it.

\section{Patient P10, MDD patient}

Three new factors emerged from the interview data that were not identified in the literature.

Nature of the clinician (87 references, 14/17 participants), with subfactors caring clinical team (24 references, 7/17 participants), level of involvement (23 references, 8/17 participants), and expertise (17 references, $6 / 17$ participants) emerged as a new top-tier factor:

Two pathways determine the decision of whether to go ahead or not. One is the more informational knowledge pathway and the other is much more emotional. In terms of the informational pathway, I was aware of the literature on $D B S$ for OCD [but not aware of the literature on MRgFUS for OCD]... But the other, though, the other has to do with trust, and I think that's a much weightier component in the decision-making.

\section{Patient P11, OCD \& MDD Patient}

The potential to enroll in future trials (18 references, 6/17 patients) was cited as a potential indirect benefit that provided further motivation to participate in a FIH trial:

If there's a study then let's just participate. Even though we knew that...there wasn't going to be like oh, all of a sudden he was back to normal. That was never the understanding that that would happen. And that the first study was really to find out whether they're onto something and whether they were going to go for, you know, a phase two.

Patient P5, AD Patient

Patient interest to be involved in the endeavor emerged as a motivating factor:

I mean I thought it was something out of Star Trek or Star Wars. It was just totally fascinating to us.

\section{Patient P6, ALS Patient}

Finally, participants expressed an interest in more robust decision support to navigate the translational process for FIH neuro trials. We noted many references to the benefit of peer support and the value in speaking with other patients, both those who have previously undergone the intervention and those who have not but suffer from the same condition.

\section{Discussion}

Through an extensive analysis of the FIH literature, we identified 224 journal papers from nine disciplines that revealed 130 key factors relevant to FIH decision-making. Considerations relating to the adequacy of preclinical evidence and FIH trial design were shared dominantly between neuro* and non-neuro literature. Factors identified as especially relevant to the translation of neurointerventions were scientific rationale, target organ and function, use of animal models, and illness prevalence.

Findings from the interviews supplement the literature analysis and emphasize the nature of the intervention, nature of the patient, and nature of the condition for FIH readiness. They further reveal different perceptions of invasiveness between clinicians and patients, and different motivations for participation in FIH clinical trials among patients with neurodegenerative and psychiatric conditions.

We anticipated that the neuro* literature would feature more discussion of moral/ontological factors such as impact on personhood, sense of self, and impact on autonomy than the non-neuro literature, but we found instead that neuro* ontological considerations were matched by moral concerns in non-neuro literature largely relating to the use of embryonic stem cells, on the one hand, and to the transformation of human nature on to the other. However, while moral/ontological considerations were no more frequently addressed in the neuro* literature than non-neuro literature, ontological factors (such as impact on personhood/ sense of self and impact on autonomy) were discussed extensively 
in interviews by patients undergoing interventions for psychiatric conditions.

Differences in the evaluation of the invasiveness of MRgFUS between patients and clinicians are a key finding. Despite clinicians' reported efforts to convey MRgFUS as an invasive procedure, many patients still described the procedure as noninvasive. Either clinician messaging is failing, patients have inherently different understandings of the concept of invasiveness, or both. When probed for their own personal definitions of invasiveness, two main themes emerged: the physical opening of the body as a requisite for an intervention to be invasive, and the irreversibility of an intervention. Close attention to this factor is essential in the context of informed consent for FIH trials.

The difference in the motivation to participate in clinical trials reported by patients with neurodegenerative conditions and patients with psychiatric conditions may be attributed to the differing goals of the trials. While the aim for MRgFUS for psychiatric conditions was to mimic the benefit of traditional psychiatric neurosurgeries without the risks associated with open surgery, the aim of the neurodegenerative trial was to study the safety and feasibility of opening the BBB. As such, no direct benefit in the form of symptom improvement could reasonably be expected for the neurodegenerative patients.

This consideration could explain another factor that emerged from the interview data: the possibility that enrollment in a therapeutic FIH trial may be a motivation for participation. Researchers should give considered attention to this potential benefit in their informed consent process and research materials prepared for prospective participants.

Finally, the nature of the clinician was a top-tier factor that emerged from the interview data. It was cited in the interviews more than benefit, adequacy of preclinical evidence, and trial design. Trustworthiness, expertise, openness, and caring characterized the features of clinicians and clinical teams that gave patients the confidence to consider and undergo an untested intervention.

\section{LIMITATIONS}

\section{Scoping Review}

We used a rich coding strategy to code the papers so that articles could be coded for multiple categories, subcategories, or factors relevant to FIH-decision-making, as appropriate. This was a necessary methodological strategy that has an effect on the reported numbers.

\section{Semi-Structured Interviews}

The small sample size in this study makes it difficult to generalize results to entire populations. This study was designed to be a small, qualitative examination of the lived experiences of a specific population, and should be interpreted as such. It is difficult to compare between the different neurogenerative and neuropsychiatric conditions examined in this study (i.e., AD and psychiatric conditions) given their inherently different natures, and as such the findings should not be generalized across conditions. Demographic data aside from patient conditions and clinician specialties were not collected, resulting in a less complete picture of the phenomenon at hand. However, it should be stated that clinical trial participants interviewed here represented adult patient populations only; a parallel discussion in pediatric populations would have unique and complex ethical considerations. In addition, the small sample sizes and the fact that interviews were conducted at only one institution meant that reporting such information could jeopardize the anonymity of the patients. Finally, only patients who chose to undergo the novel MRgFUS over another, more standard therapy or no treatment at all were enrolled in our study. It would be interesting to see what factors determined a patient's nonparticipation. Further work could benefit from a larger sample that also contains participants who declined the novel intervention.

\section{Conclusion}

This work sought to shed light on critical factors in determining the readiness of a novel FIH neuromodulatory intervention using MRgFUS as a case study. Results indicate different priorities, values, and understandings between clinicians and patients, and patients affected by different neurologic conditions. Such findings must be addressed in consent strategies for FIH trials involving people affected by neurologic and other devastating conditions, development of guidance documents for clinicians, and support materials for future patients.

\section{ACKNOWLedgements}

The authors would like to acknowledge and wholeheartedly thank the participants who agreed to be interviewed. We would also like to acknowledge Sunnybrook Health Sciences Center for allowing us to use their facilities and PCNEC for the initiation and funding of this project.

\section{FUNDING}

This work was supported by Sunnybrook Health Sciences Centre through a contract to Neuroethics Canada at the University called the Pan-Canadian Neurotechnology Collaboration (PCNEC). JI is Canada Research Chair in Neuroethics; PJM is Alcan Chair in Neurosciences at UBC.

\section{CONFLict of INTEREST}

None of the authors have financial conflicts of interest to disclose. JI and PJM are recipients of a grant from the NIH Brain Initiative.

\section{Author CONTRIButions}

ICM conceptualized the study design and led data collection, data analysis, and writing of the manuscript. NM assisted with data collection, data analysis, and manuscript editing. AB facilitated data collection and development of research materials. NL contributed to project inception and conceptualization, study design, and manuscript editing. PJM contributed to project inception and conceptualization and manuscript editing. JI was responsible for all aspects of project inception and conceptualization, study design, and manuscript writing and editing.

\section{SUPPLEMENTARY MATERIAI}

To view supplementary material for this article, please visit https://doi.org/10.1017/cjn.2020.113 


\section{REFERENCES}

1. Genske A, Engel-Glatter S. Rethinking risk assessment for emerging technology first-in-human trials. Med Health Care Philos. 2016;19(1):125-39.

2. McDonald PJ, Kulkarni AV, Farrokhyar F, Bhandari M. Ethical issues in surgical research. Can J Surg. 2010;53(2):4.

3. Lipsman N, Bernstein M, Lozano AM. Criteria for the ethical conduct of psychiatric neurosurgery clinical trials. Neurosurg Focus. 2010;29(2):E9.

4. McCulloch P, Altman DG, Campbell WB, et al. No surgical innovation without evaluation: the IDEAL recommendations. Lancet. 2009;374(9695):1105-12.

5. McDonald PJ, Lau C, Coates McCall I, Lipsman N, Illes J. Regulatory oversights for implantable neurodevices. Lancet Neurol. 2019;18(10):913.

6. Barazzetti G, Hurst SA, Mauron A. Adapting preclinical benchmarks for first-in-human trials of human embryonic stem cell-based therapies: adapting preclinical benchmarks for hESC therapies. Stem Cells Transl Med. 2016;5(8):1058-66.

7. Kimmelman J. A theoretical framework for early human studies: uncertainty, intervention ensembles, and boundaries. Trials. 2012;13(1):173.

8. World Medical Association. World medical association declaration of Helsinki: ethical principles for medical research involving human subjects. J Am Med Assoc. 2013;310(20):2191-4.

9. Chapman AR. Addressing the ethical challenges of first in-human trials. J Clin Res Bioeth. 2011;2(4). doi:10.4172/2155-9627. 1000113

10. Dresser R. First-in-human HIV-remission studies: reducing and justifying risk. J Med Ethics. 2017;43(2):78-81.

11. Dresser R. Building an ethical foundation for first-in-human nanotrials. J Law Med Ethics. 2012;40(4):802-8.

12. Kimmelman J. Gene transfer and the ethics of first-in-human research. Cambridge University Press; 2010. doi:10.1017/ CBO9780511642364

13. Margo CE. When is surgery research? Towards an operational definition of human research. J Med Ethics. 2001;27(1):40-3.

14. Specker Sullivan L, Illes J. Ethics in published brain-computer interface research. J Neural Eng. 2018;15(1):013001.

15. Weisberg DS, Keil FC, Goodstein J, Rawson E, Gray JR. The seductive allure of neuroscience explanations. J Cogn Neurosci. 2008;20(3):470-7.

16. Minielly N, Hrincu V, Illes J. Privacy challenges to the democratization of brain data. iScience. 2020;23(6):101134.
17. Bernstein M, Bampoe J. Surgical innovation or surgical evolution: an ethical and practical guide to handling novel neurosurgical procedures. J Neurosurg. 2004;100(1):2-7.

18. Klein E. Eloquent brain, ethical challenges: functional brain mapping in neurosurgery. Semin Ultrasound CT MRI. 2015;36(3): $291-5$.

19. Mainprize T, Lipsman N, Huang Y, et al. Blood-brain barrier opening in primary brain tumors with non-invasive MR-guided focused ultrasound: a clinical safety and feasibility study. Sci Rep. 2019;9(1):321.

20. Lipsman N, Meng Y, Bethune AJ, et al. Blood-brain barrier opening in Alzheimer's disease using MR-guided focused ultrasound. Nat Commun. 2018;9(1):2336.

21. Abrahao A, Meng Y, Llinas M, et al. First-in-human trial of blood-brain barrier opening in amyotrophic lateral sclerosis using MR-guided focused ultrasound. Nat Commun. 2019;10(1):4373.

22. Arksey H, O'Malley L. Scoping studies: towards a methodological framework. Int J Soc Res Methodol. 2005;8(1):19-32.

23. Sandelowski M. Whatever happened to qualitative description? Res Nurs Health. 2000;23(4):334-40.

24. Neergaard MA, Olesen F, Andersen RS, Sondergaard J. Qualitative description - the poor cousin of health research? BMC Med Res Methodol. 2009;9(1):52.

25. Hsieh H-F, Shannon SE. Three approaches to qualitative content analysis. Qual Health Res. 2005;15(9):1277-88.

26. Kyngas H, Vanhanen L. Content analysis as a research method. Hoitotiede. 1999;11:3-12.

27. King NMP. Nanomedicine first-in-human research: challenges for informed consent. J Law Med Ethics. 2012;40(4):823-30.

28. Tammara B, Raje S, McKeand W, Korth-Bradley JM. Translational research: preclinical to healthy volunteer to patient. In: Bhattachar SN, Morrison JS, Mudra DR, Bender DM, editors. Translating molecules into medicines. Cham: Springer International Publishing; 2017; pp. 341-72.

29. Menalled L, Brunner D. Animal models of Huntington's disease for translation to the clinic: best practices: ANIMAL MODELS OF HD. Mov Disord. 2014;29(11):1375-90.

30. Satalkar P, Elger BS, Hunziker P, Shaw D. Challenges of clinical translation in nanomedicine: a qualitative study. Nanomedicine Nanotechnol Biol Med. 2016;12(4):893-900.

31. Kimmelman J. Ethics, ambiguity aversion, and the review of complex translational clinical trials. Bioethics. 2012;26(5): $242-50$. 